\title{
Mechanism of floral scent production in Osmanthus fragrans and the production and regulation of its key floral constituents, $\beta$-ionone and linalool
}

\author{
Yuanji Han $\mathbb{D}^{1}$, Hongyun Wang ${ }^{1}$, Xiaodan Wang ${ }^{1}, \mathrm{Ke} \mathrm{Li}^{1}$, Meifang Dong ${ }^{1}$, Yong Li', Qian Zhu ${ }^{1}$ and Fude Shang ${ }^{1}$
}

\begin{abstract}
Sweet osmanthus (Osmanthus fragrans Lour.) is among the top ten most well-known flowers in China and is recognized as both an aromatic plant and ornamental flower. Here, manual sectioning, scanning electron microscopy, and transmission electron microscopy of sweet osmanthus petals revealed that large amounts of lipids are present inside the petal cells and on the cell surfaces. However, no secretory structures were observed. Instead, the petal cells protrude slightly outward, and the surfaces of the cells are adorned with highly regular brush-shaped hairs. The surfaces of the 'Yingui' petals possessed mostly curled and more numerous hairs, whereas the 'Dangui' petals possessed fewer brush-shaped and more sparsely arranged hairs. In addition, many granular substances were attached to the brush-shaped hairs, and the granules were denser on the hairs of the 'Yingui' petals compared to the hairs on the 'Dangui' petals. Furthermore, 35 aromatic components in the 'Yingui' petals and 30 aromatic components in the 'Dangui' petals were detected via GC-MS. The main aromatic component of the 'Yingui' petals was $\beta$-ionone, whereas that of the 'Dangui' petals was linalool and its oxides. Transcriptome sequencing and GRT-PCR indicated that the high $\beta$-ionone content in the 'Yingui' petals was due to the overexpression of CCD1 and CCD4 and that the high linalool content in the 'Dangui' petals was due to the overexpression of MECS, HDR, IDII, and LIS1, which function upstream of the linalool synthetic pathway. In particular, the expression levels of CCD4 and LIS1 were upregulated by 5.5- and 5.1fold in the 'Yingui' and 'Dangui' petals, respectively. One transcription factor (ERF61) was cloned and named, and the expression pattern of ERF61 in sweet osmanthus petals was found to be generally consistent with that of CCD4. Tobacco transformation experiments, yeast one-hybrid experiments, and electrophoretic mobility shift assays indicated that ERF61 binds to the CCD4 promoter and stimulates CCD4 expression, thereby regulating the synthesis of $\beta$-ionone in sweet osmanthus petals.
\end{abstract}

\section{Introduction}

Floral fragrance, color, shape, surface structure, and nectar glands can affect the interactions of higher plants with pollinators, and floral fragrance, in particular, can

Correspondence: Yuanji Han (wolianghan@163.com) or

Fude Shang (fudeshang@henu.edu.cn)

${ }^{1}$ School of Life Sciences, State Key Laboratory of Cotton Biology, State Key Laboratory of Crop Stress Adaptation and Improvement, Key Laboratory of Plant Stress Biology, Laboratory of Plant Germplasm and Genetic Engineering, Henan University, Kaifeng 475004 Henan, China

These authors contributed equally: Yuanji Han, Hongyun Wang also function to deter herbivores and attract natural enemies $^{1,2}$. Floral scents generally contain complex volatile components, such as terpenoids and terpenoid derivatives, aromatic components, fatty acid derivatives, and other low-molecular-weight compounds, and the production and release of volatile oils from plant tissues are often associated with specialized tissues and organs, such as glandular hairs, oil glands, or resin tubes ${ }^{2-4}$. In some plants, such as members of Asclepiadaceae, Aristolochiaceae, Araceae, Burmanniaceae, and Orchidaceae,

\section{(c) The Author(s) 2019}

(c) (i) Open Access This article is licensed under a Creative Commons Attribution 4.0 International License, which permits use, sharing, adaptation, distribution and reproduction c. in any medium or format, as long as you give appropriate credit to the original author(s) and the source, provide a link to the Creative Commons license, and indicate if changes were made. The images or other third party material in this article are included in the article's Creative Commons license, unless indicated otherwise in a credit line to the material. If material is not included in the article's Creative Commons license and your intended use is not permitted by statutory regulation or exceeds the permitted use, you will need to obtain permission directly from the copyright holder. To view a copy of this license, visit http://creativecommons.org/licenses/by/4.0/. 
floral scents have been reported to originate from unique "aromatic glands" ${ }^{-10}$.

In plants with aromatic glands, different parts of the flower can differentiate into flap-, cilia-, or hair-shaped glands that function in the production and emission of aromatic substances ${ }^{9,10}$. After volatile oils are produced in the secretory cells of aromatic glands, the oils are usually immediately released and disperse within a short period of time $^{5}$. However, such oils can also form intracellular droplets. The mechanisms underlying floral scent production likely vary among plants and remain poorly understood.

$O$. fragrans is valued for its beauty and fragrance. The fragrance of the flower contains as many as 30 potentially exploitable chemical substances ${ }^{11-13}$. The plant has been cultivated for more than 2500 years in China and is among the top ten most well-known flowers in China. The flowering period, floral color, and other characteristics are used to classify sweet osmanthus cultivars into four groups: Yingui, Jingui, Dangui, and Sijigui ${ }^{14,15}$. Flowers in the Yingui group are relatively light in color (i.e., pale to medium yellow), whereas those of the Dangui group are relatively deep in color (i.e., orange to orangered), and this difference is mainly attributed to differences in the levels of carotenoids, especially $\beta$-carotene ${ }^{16,17}$. The protein encoded by $C C D 1$ in sweet osmanthus is capable of cleaving carotenoids into ionones (e.g., $\alpha$-carotene to $\alpha$ ionone and $\beta$-ionone and $\beta$-carotene to $\beta$-ionone $)^{18,19}$, whereas the protein encoded by CCD4 can cleave $\beta$-carotene into $\beta$-ionone ${ }^{20}$. Previous studies of sweet osmanthus have also reported that the WRKY1 transcription factor regulates the expression of CCD4 and might therefore regulate the cleavage of carotenoids and the synthesis of $\beta$-ionone, a key aromatic component ${ }^{21}$. Our previous study also revealed that the CCD4 promoter region of sweet osmanthus contains multiple ERF transcription factor-binding elements and might therefore be regulated by an ERF transcription factor.

AP2/ERF transcription factors are a large family of transcription factors in plants that are named for their AP2/ERF domain, which is composed of 60-70 amino acids. They are generally separated into three subfamilies (AP2, ERF, and RAV) on the basis of sequence similarity and number of AP2/ERF domains ${ }^{22}$. ERF subfamily members contain a single AP2/ERF domain and can be further divided into two large subfamilies: the ERF subfamily and the CBF/DREB subfamily ${ }^{23}$. The Arabidopsis thaliana AP2/ERF transcription factor RAV1 recognizes CAACA and CACCTG elements, thereby regulating the expression of corresponding genes ${ }^{24}$. CARAV1, a new RAV gene, was isolated from pepper leaves that were infected with Xanthomonas campestris pv. vesicatoria. As in A. thaliana, the CARAV1 protein is able to recognize CAACA and CACCTG elements ${ }^{25}$, thereby regulating the expression of corresponding genes. In tobacco, an ERF transcription factor located at the NIC-2 locus is required to induce the jasmonate-mediated activation of nicotine biosynthesis ${ }^{26}$, and in Catharanthus roseus ${ }^{27}$, the jasmonate-responsive genes ORCA2 and ORCA3 control the expression of strictosidine synthase, which is required for the biosynthesis of terpenoid indole alkaloids.

O. fragrans is famous for its unique fragrance. Researching the production and regulatory mechanisms of the crucial floral scent components in sweet osmanthus could enrich our foundational knowledge of the molecular biology of sweet osmanthus, provide a theoretical basis for the utilization of primary floral scent components in sweet osmanthus, and improve the breeding of sweet osmanthus cultivars.

\section{Materials and methods \\ Materials}

Freshly cut flowering branches of O. fragrans 'Baijie' (Yingui group) and 'Chenghong Dangui' (Dangui group) were incubated at a constant temperature $\left(22^{\circ} \mathrm{C}\right)$ and relative humidity $(70 \%)$ under a $12 \mathrm{~h}: 12 \mathrm{~h}$ light:dark cycle $\left(80 \mu \mathrm{mol} \mathrm{m}^{-2} \mathrm{~s}^{-1}\right)^{21,28}$. Flowers at the full flowering stage were collected for analysis.

\section{Manual section detection}

Fresh sweet osmanthus petals were manually sectioned, stained using 1\% Sudan Black, and then observed and photographed using an Olympus BX60 microscope (Olympus, Tokyo, Japan).

\section{Scanning electron microscopy}

Fresh petals were separated into two parts. One part was fixed in $2 \%$ glutaraldehyde for $3-5 \mathrm{~h}$, washed using $0.1 \mathrm{M}$ phosphate buffer, dehydrated using an ethanol gradient (30\%, 50\%, $70 \%$, and $100 \%$, successively), transferred to isoamyl acetate, and then subjected to critical-point drying using carbon dioxide. After being coated with gold, the petals were observed and photographed using a PHILIPS XL30ESEM environmental scanning electron microscope (ESEM) (Philips, Eindhoven, Netherlands). Meanwhile, the second part was directly adhered to a sample platform, subjected to ultralow-temperature freezing and coating with gold, transferred to a cold stage, and observed and photographed using a HITACHI S-3400N cryo-scanning electron microscope (cryo-SEM) (Hitachi, Tokyo, Japan).

\section{Transmission electron microscopy}

Fresh sweet osmanthus petals were cut into $2 \mathrm{~mm} \times$ $5 \mathrm{~mm}$ small blocks, fixed overnight in $1.25 \%$ paraformaldehyde and $1.25 \%$ glutaraldehyde (prepared using $0.1 \mathrm{M}$ phosphate buffer, $\mathrm{pH} 7.0$ ), washed using $0.1 \mathrm{M}$ phosphate buffer, fixed in $1 \%$ osmic acid for $2.5 \mathrm{~h}$, washed 
again using $0.1 \mathrm{M}$ phosphate buffer, dehydrated using an ethanol gradient (30\%, 50\%, 70\%, and 100\%, successively), and then embedded in Epon812 epoxy resin. The embedded petals were then sectioned using a Leica ULTRACUT R ultramicrotome (Leica, Heidelberg, Germany), stained using uranyl acetate and lead citrate for 5-6 min, and observed and photographed using a JEM 100CX-II transmission electron microscope (TEM) (Jeol, Tokyo, Japan).

\section{Gas chromatography-mass spectrometer (GC-MS) analysis of sweet osmanthus petals}

Fifty 'Yingui' and 50 'Dangui' petals were separately sealed in $15 \mathrm{~mL}$ extraction flasks. After a 20-min equilibration period, a solid phase microextraction head was inserted, and after $40 \mathrm{~min}$ of headspace adsorption at $50-55^{\circ} \mathrm{C}$, the extraction head was removed, and the sample was loaded into the inlet port of the GC-MS system.

The chromatography conditions were as follows: TR5 MS $(30 \mathrm{~m} \times 0.25 \mathrm{~mm} \times 0.25 \mu \mathrm{m})$ elastic quartz capillary column; a column flow of $1.1 \mathrm{~mL} / \mathrm{min}$; a temperature program of $50^{\circ} \mathrm{C}$ for $2 \mathrm{~min}$, an increase of $5{ }^{\circ} \mathrm{C} / \mathrm{min}$ until reaching $120^{\circ} \mathrm{C}, 120^{\circ} \mathrm{C}$ for $2 \mathrm{~min}$, an increase of $7^{\circ} \mathrm{C} / \mathrm{min}$ until reaching $220^{\circ} \mathrm{C}$, and $220^{\circ} \mathrm{C}$ for $10 \mathrm{~min}$; the carrier gas was highly pure helium (99.999\%); and the splitless sample loading method.

The mass spectrometry conditions were as follows: Ei ion source; an ion trap temperature of $150^{\circ} \mathrm{C}$; a manifold temperature of $40^{\circ} \mathrm{C}$, a GC-MS transmission temperature $(I / F)$ of $250^{\circ} \mathrm{C}$; a mass scanning range $(\mathrm{m} / z)$ of $35-600 \mathrm{u}$; an ionization energy of $70 \mathrm{eV}$; an emission current of $150 \mu \mathrm{A}$; and a detector voltage of $350 \mathrm{~V}$. The experimental conditions were strictly controlled, and parallel experiments were performed in triplicate under identical conditions to analyze the accuracy and reliability of the solid phase microextraction (SPME) GC/MS data.

\section{RNA sequencing}

RNA sequencing (RNA-Seq) was performed by BioMarker Technology (Beijing, China). The mRNA was isolated from petals, cut into shorter fragments, and ligated with adaptors. The first cDNA strand was produced using random primers and reverse transcription kits. The second cDNA strand was synthesized using DNA polymerase I, and adapter oligonucleotides with a hairpin loop structure were ligated to prepare them for hybridization. cDNA fragments of $150-250 \mathrm{bp}$ were preferentially selected for sequencing on the Illumina HiSeq 2500 (Illumina, San Diego, USA) sequencing platform.

The expression levels of unigenes were calculated using the fragments per kilobase million (FPKM) method. Gene expression levels of differentially expressed genes (DEGs) were computed by the following formula: FPKM $=\mathrm{cDNA}$ fragments/[mapped fragments (millions) $\times$ transcript
Length (kb)]. The FPKM results can be directly used to compare gene expression levels within differential samples.

\section{RNA extraction and CDNA cloning of the ERF61 gene}

Total RNA was extracted from flower petals using a MiniBEST universal RNA extraction kit (TaKaRa, Dalian, China) according to the manufacturer's instructions. RNA quality and integrity were assessed using formamide denaturing gel electrophoresis, and RNA quantity was measured using spectrophotometry ${ }^{29}$. cDNA was then synthesized using a Superscript preamplification kit (Invitrogen, California, USA) according to the manufacturer's instructions.

Forward (ERF61f1) and reverse (ERF61r1) primers were designed and used to amplify the full-length ERF61 cDNA sequence under the following PCR amplification conditions: $94{ }^{\circ} \mathrm{C}$ for $4 \mathrm{~min}$ followed by 33 cycles of $94{ }^{\circ} \mathrm{C}$ for $30 \mathrm{~s}, 55^{\circ} \mathrm{C}$ for $45 \mathrm{~s}$, and $72^{\circ} \mathrm{C}$ for $60 \mathrm{~s}$. The PCR product was cloned into the pMD19-T vector (TaKaRa) and sequenced. Next, the $O$. fragrans ERF61 (OfERF61) amino acid sequence was compared to ERF sequences from other plant species. More specifically, the sequences were aligned using Clustal W version 1.83, phylogenetic analysis was performed using MEGA 4.1, and a neighborjoining (NJ) tree was constructed using the distance matrix computed by MEGA.

\section{Reverse transcriptase-PCR, quantitative real-time PCR analysis}

RNA was extracted from the petals of the 'Dangui' and 'Yingui' cultivars at the xiangyan stage (S1), initial flowering stage (S2), full flowering stage (S3), and late flowering stage (S4). Reverse transcriptase PCR (RT-PCR) and quantitative real-time PCR (qRT-PCR) primers are shown in Supplementary Table 1. The expression level of ACTIN was used as a reference, and RT-PCR amplification was performed using the following conditions: $94{ }^{\circ} \mathrm{C}$ for $4 \mathrm{~min}$ and 34 cycles of $94{ }^{\circ} \mathrm{C}$ for $30 \mathrm{~s}, 57^{\circ} \mathrm{C}$ for $30 \mathrm{~s}$, and $72{ }^{\circ} \mathrm{C}$ for $45 \mathrm{~s}$. The reactions were performed in triplicate. qRT-PCR was performed using a Roche LightCycler 480II detection system, and each $20 \mu \mathrm{L}$ reaction contained $10 \mu \mathrm{L}$ SYBR Premix Ex Taq mix (Takara). Relative expression levels were calculated using the $2^{-\Delta \Delta \mathrm{Ct}}$ method, and each analysis included three to five replicates.

\section{Transient transformation in tobacco leaves}

A CCD $4_{\text {pro: }}$ :GUS vector was constructed by using PCR and specific primers (CCD4f and CCD4r) to amplify a 976-bp OfCCD4 promoter region (accession no. KF701121) and clone it into the pCAMBIA1391 vector. Meanwhile, the OfERF61 cDNA sequence was amplified via PCR using gene-specific primers (ERF61f2 and ERF61f2) and cloned into the pHBT vector to construct the $35 \mathrm{~S}_{\text {pro: }}: E R F 61$ vector. More specifically, the CCD $4_{\text {pro: }}$ : 


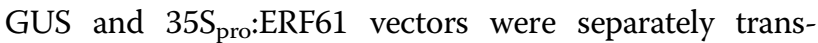
formed into A. tumefaciens strain EHA105 using the freeze-thaw method, and EHA105 strains that contained the CCD $4_{\text {pro: }}$ GUS and 35S:ERF61 vectors were mixed at a $1: 1$ volume ratio, incubated at $25^{\circ} \mathrm{C}$ for $2-4 \mathrm{~h}$, and then cotransformed by transferring the vectors via a syringe onto the abaxial surface of fully expanded tobacco leaves. Leaves coinfiltrated with Agrobacteria carrying 35S:GFP and $\mathrm{CCD} 4_{\text {Pro: }}$ GUS vectors were used as controls. GUS activity was assayed by incubating tissue samples overnight at $37{ }^{\circ} \mathrm{C}$ in a $0.1 \%(\mathrm{w} / \mathrm{v}) \mathrm{X}$-Gluc solution that contained $50 \mathrm{mM}$ sodium phosphate buffer $(\mathrm{pH} 7.2), 0.1 \%(\mathrm{w} / \mathrm{v})$ Triton X-100, $2 \mathrm{mM} \mathrm{K}_{3} \mathrm{Fe}(\mathrm{CN})_{6}, 2 \mathrm{mM} \mathrm{K}_{4}\left[\mathrm{Fe}(\mathrm{CN})_{6}\right] \cdot 3 \mathrm{H}_{2} \mathrm{O}$, and $10 \mathrm{mM}$ EDTA. After staining, green tissues were bleached by soaking them for a few hours in $50 \%$ ethanol and were then washed using $70 \%$ ethanol.

\section{Yeast one-hybrid assay}

The promoter region of the OfCCD4 gene contains several CAACA elements (Supplementary Fig. 1), which are the binding sites of ERF transcription factors. Four copies of the CAACA element from the OfCCD4 promoter were artificially synthesized into double-stranded DNA (C1), and both ends of the strand were modified with EcoRI and $\mathrm{XhoI}$ restriction endonuclease sites. The $\mathrm{C} 1$ strand was then annealed to EcoRI- and XhoI-digested pLacZi vectors to form the pLacZi-C1 recombinant vector. The OfERF61 cDNA sequence was amplified using a $X h o I$ site-containing forward primer (ERF61f3) and a BamHI site-containing reverse primer (ERF61r3), purified, digested using BamHI and XhoI, and then annealed to a XhoI- and BamHIdigested pJG vector to form the pJG-ERF61 recombinant vector. Next, the pLacZi-C1 and pJG vector combination and pLacZi-C1 and pJG-ERF61 vector combination were cotransformed into YM4271 yeast cells. The resulting yeast cells were cultured in SD-Trp-Ura double-deficiency medium for 2-3 days, transferred to a nylon membrane, subjected to 2-3 freeze-thaw cycles in liquid nitrogen, and then incubated in staining buffer/X-gal solution for $30 \mathrm{~min}$ to $8 \mathrm{~h}$ at $30^{\circ} \mathrm{C}$.

\section{In vitro ERF61 protein synthesis and electrophoretic mobility shift assay}

The ERF61 cDNA sequence was amplified using an EcoRI site-containing forward primer (ERF61f4) and an XhoI site-containing reverse primer (ERF61r4), ligated into an EcoRI- and XhoI-digested pET-30a-c vector, and transformed into competent Escherichia coli BL21(DE3) cells. Protein expression and purification were then performed as described by Han et al. ${ }^{21}$, and the purified ERF61 protein was used for electrophoretic mobility shift assay (EMSA). More specifically, $3^{\prime}$ biotin-labeled sense oligonucleotides that contained both the CAACA element of the $O f C C D 4$ promoter and the corresponding antisense strand sequence were synthesized; the two oligonucleotides were annealed to form double-stranded DNA, and EMSA was performed using the LightShift Chemiluminescent EMSA Kit (Thermo Fisher, Waltham, USA) and Chemiluminescent Nucleic Acid Detection Module (Thermo Fisher, Waltham, USA), according to the manufacturer's instructions.

\section{Results}

\section{Structure of sweet osmanthus petals}

Sudan Blackdye is an efficient dye for lipid stains and dyes lipid granules in the cell a grayish-black, brownblack, or black color ${ }^{30-33}$. In this study, after Sudan Black staining, the petal cells appeared brown-black, and brown-black granules could be observed inside the cells (Fig. 1), which indicated that the petal cells contained large amounts of lipids.

ESEM and cryo-SEM revealed an absence of secretory structures on the surface of the sweet osmanthus petals. Instead, the petal cells protruded slightly outward, and the cell surfaces of both cultivars were adorned with extremely regular brush-like strips known as brush-shaped hairs (Fig. 2a-h). However, the 'Yingui' petal surfaces possessed more densely arranged brush-shaped hairs that protruded significantly and were mostly curled (Fig. $2 \mathrm{e}-\mathrm{h}$ ), whereas the 'Dangui' petal surfaces possessed more sparsely arranged brush-shaped hairs that did not protrude and were nearly parallel in arrangement (Fig. 2a-d). Observation of the petal epidermis at high magnification also revealed that large numbers of granules were attached to the brush-shaped hairs, with some granules located between strips, and the granules on the 'Yingui' cultivar hairs were far greater in density and quantity than those of the 'Dangui' cultivar (Fig. 2c, d, g, h). Furthermore, the cryo-SEM results for the other three Dangui group cultivars ('Zhushagui', 'Yingye Dangui', and 'Zuijihong') (Fig. 2i-k) and the three Yingui group cultivars (' $\mathrm{Zi}$ Yingui', 'Zaohuang', and 'Wan Yingui') (Fig. 2l-n) were consistent with those of the two aforementioned cultivars.

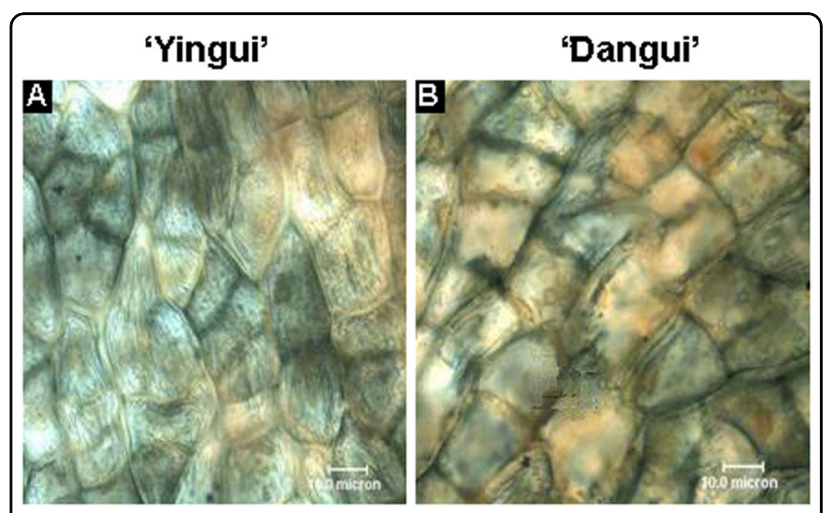

Fig. 1 Manual section results of flower petals. a 'Yingui' and $\mathbf{b}$ 'Dangui' 


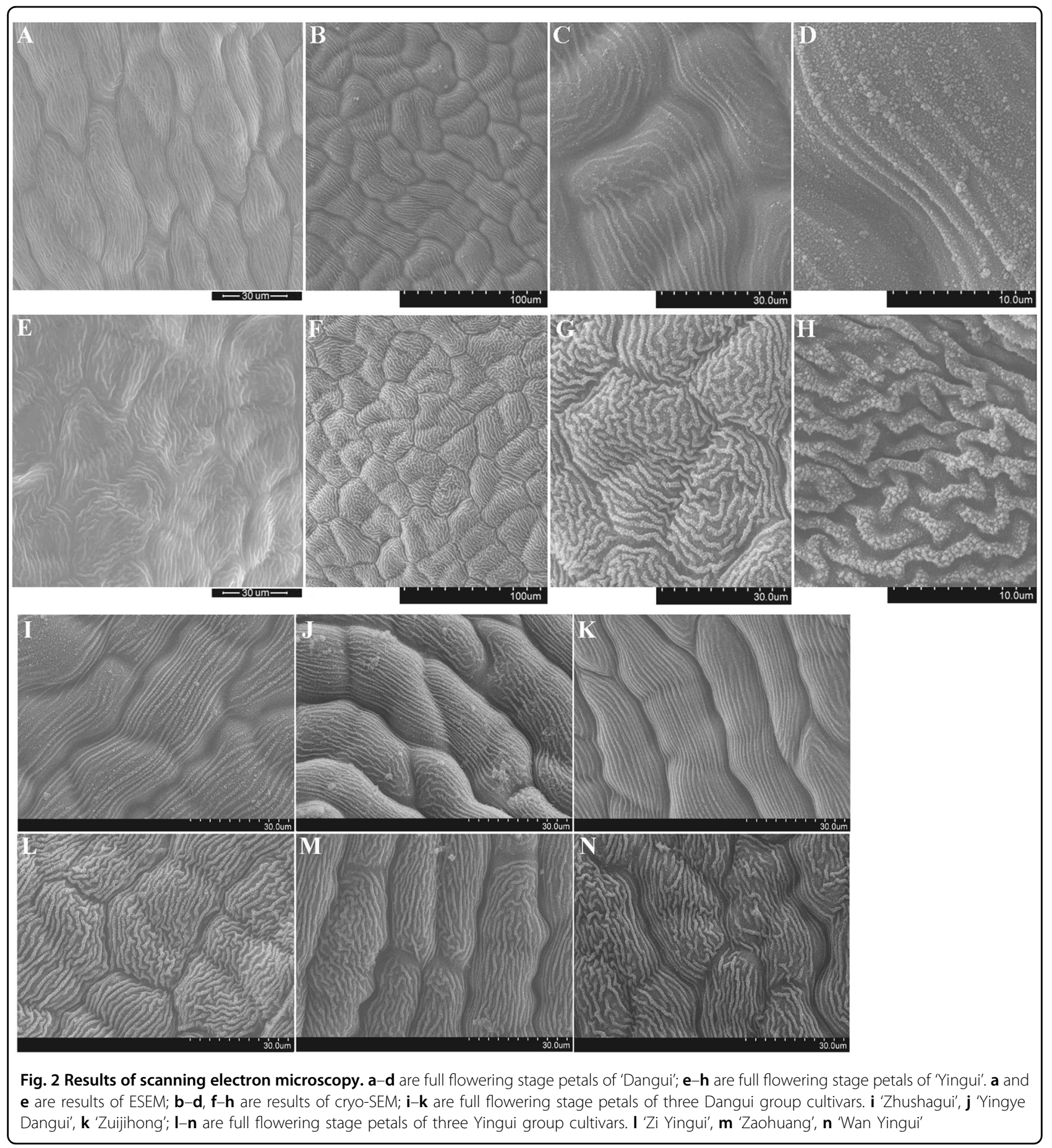

The differences in the epidermal cell morphology, density of brush-shaped hairs, and quantity of granules could be related to the different floral scent components and contents of the flower petals in the two cultivars.

TEM confirmed that the sweet osmanthus petals lacked secretory structures and revealed that most of the petal cells contained electron-dense osmiophilic granules (Fig. $3 a-f)$. Electron microscopy analysis revealed that the osmiophilic granule density on the surface and interior of the petal cells was higher in 'Yingui' compared to the 'Dangui' cultivar (Fig. 3b, e).

\section{Volatile aroma compounds of sweet osmanthus petals}

GC-MS analysis revealed that 30 and 35 floral scent components were detected in the 'Dangui' and 'Yingui' petals, respectively, and two components, $\beta$-ionone and 


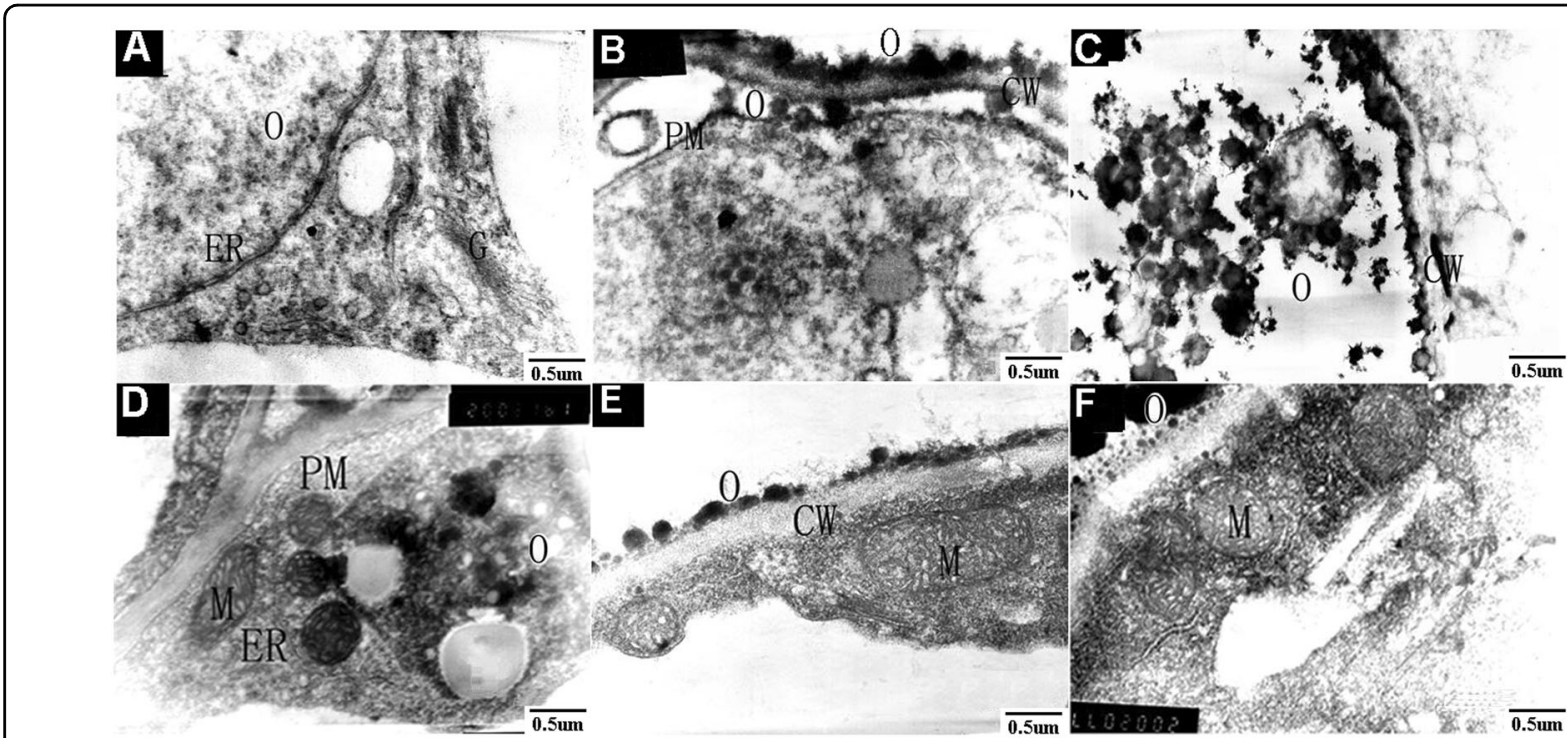

Fig. 3 Results of transmission electron microscopy. a-c are ground petal tissues of 'Yingui' in the full flowering stage; $\mathbf{d}$-f are ground petal tissues of 'Dangui' in the full flowering stage. ER endoplasmic reticulum, G Golgi apparatus, M mitochondrion, CW cell wall, O osmiophilic globules, PM plasma membrane

linalool, exhibited greater variation between the cultivars compared to the other scent components (Supplementary Fig. 2, Supplementary File 1). The components $\alpha$ ionone, $\beta$-ionone, and dihydro- $\beta$-ionone accounted for $0.98 \%, 0.61 \%$, and $0.38 \%$ of the total floral scent components, respectively, in the 'Dangui' cultivar but $7.7 \%$, $35.59 \%$, and $5.97 \%$, respectively, in the 'Yingui' cultivar, with $\beta$-ionone exhibiting the greatest difference between the cultivars. In contrast, both trans-linalool (19.58\%) and cis-linalool $(25.26 \%)$ were more abundant in the 'Dangui' petals than in the 'Yingui' petals $(2.31 \%$ and $2.77 \%$, respectively).

\section{Linalool and ionone metabolism-related gene expression in sweet osmanthus petals}

Transcriptome sequencing revealed that four genes involved in the linalool synthetic pathway (MECS, $H D R$, IDI1, and LIS1) were significantly upregulated in the 'Dangui' petals compared to the 'Yingui' petals (Fig. 4b, Supplementary File 2), and qRT-PCR indicated that the expression levels of MECS, HDR, IDI1, and LIS1 were 1.5-, 4.1-, 2.2-, and 5.1-fold greater in the 'Dangui' petals than in the 'Yingui' petals (Fig. 4c).

Both CCD1 and CCD4 are crucial for the cleavage of carotene into $\alpha$-ionone and $\beta$-ionone (Fig. 5a). Transcriptome sequencing indicated that the expression levels of CCD1 and CCD4 were greater in the 'Yingui' petals than in the 'Dangui' petals (Fig. 5b, Supplementary File 2), and qRT-PCR indicated that the expression of the CCD1 and $C C D 4$ genes was upregulated in the 'Yingui' petals
2.3- and 5.5-fold, respectively, compared to the expression of these genes in the 'Dangui' petals (Fig. 5c).

\section{Sequence analysis of OfERF61 CDNA}

The cDNA of OfERF61 (GenBank accession no. MK994511) appeared to be a full-length sequence of $909 \mathrm{bp}$ encoding a protein of 303 amino acid residues. Sequence alignment revealed that ERF61 shared high amino acid sequence identity with Olea europaea var. sylvestris ERF61, Solanum lycopersicum ERF61, and Glycine max ERF61 (Supplementary Fig. 3). A phylogenetic tree was constructed based on the amino acids of OfERF61 and other known ERFs from A. thaliana, and OfERF61 showed high similarity with AtERF55, AtERF59, and AtERF62 (Supplementary Fig. 4).

\section{Expression patterns of ERF61 and CCD4}

RT-PCR and qRT-PCR were used to analyze samples from the two cultivars at different flowering stages. During the S1, S3, and S4 stages, the expression of CCD4 in the 'Dangui' petals was significantly lower compared to the expression of $C C D 4$ in the 'Yingui' cultivar (Fig. 6a, b). However, the expression levels of ERF61 and CCD4 increased significantly from S1 to S4 in the 'Yingui' petals (Fig. 6c). In the 'Dangui' flower petals, the transcription levels of ERF61 and CCD4 increased significantly from S1 to $\mathrm{S} 2$, then gradually declined in $\mathrm{S} 3$, and returned to reach a peak at S4 (Fig. 6d). Although the CCD4 and ERF61 expression levels differed slightly in the two cultivars, the expression pattern of ERF61 closely resembled that of 
A

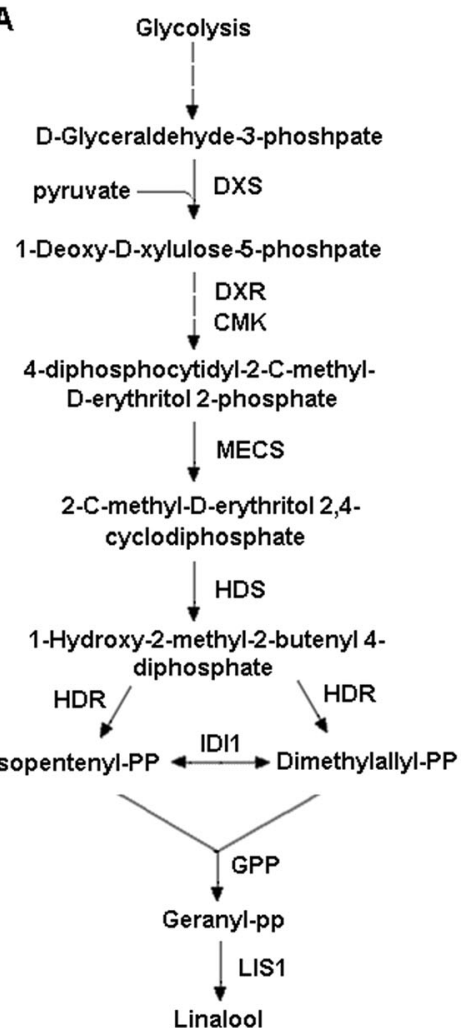

B

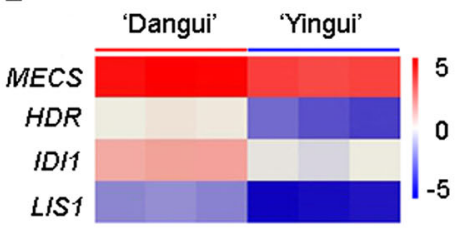

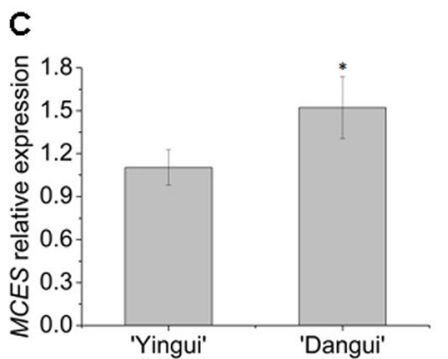

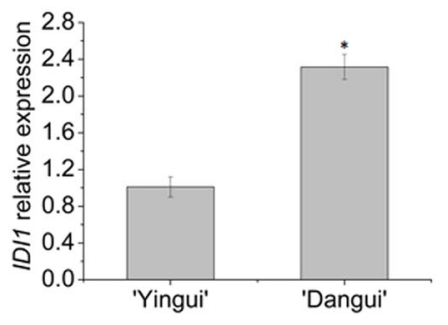

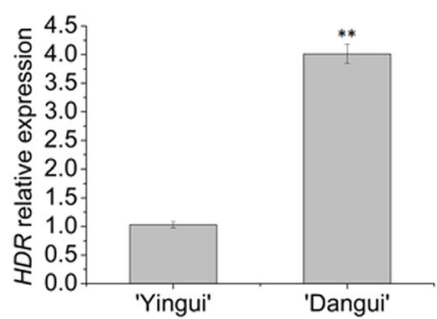

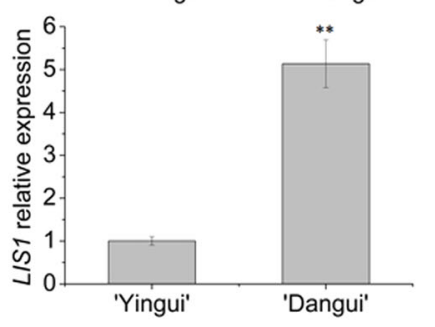

Fig. 4 Transcript levels of selected genes involved in the linalool biosynthesis pathway in the full flowering stage petals of 'Yingui' and 'Dangui'. a Linalool biosynthesis pathway. DXS 1-deoxy-D-xylulose 5-phosphate synthase, DXR 1-deoxy-D-xylulose 5-phosphate reductoisomerase, MCT 2-C-methyl-D-erythritol 4-phosphate cytidylyltransferase, CMK 4-(cytidine 5-diphospho)-2-C-methyl-D-erythritol kinase, MECS 2-C-methyl-Derythritol 2,4-cyclodiphosphate synthase, HDS 4-hydroxy-3-methylbut-2-en-1-yl diphosphate synthase, HDR 4-hydroxy-3-methylbut-2-enyl diphosphate reductase, IDI isopentenyl pyrophosphate isomerase, GPP geranyl pyrophosphate synthase, LIS linalool synthase. b MECS, HDR, IDI1, and LIS1 expression profiles from RNA-Seq. c qRT-PCR results of the MECS, HDR, IDI1, and LIS1 genes. The qRT-PCR data represent the means \pm SD of the three replicates from three independent experiments. ${ }^{*} P<0.05,{ }^{*} P<0.01$

OfCCD4 (Fig. 6a, c, d). This suggests that ERF61 may participate in $C C D 4$ regulation.

\section{Overexpression of OfERF61 upregulates the transcript levels of $\mathrm{NbCCD} 4$ in tobacco leaves}

To determine the ability of OfERF61 to upregulate transcript levels of the CCD4 gene, OfERF61 was transiently overexpressed in Nicotiana benthamiana leaves. Analyses of 35S:OfERF61-containing A. tumefaciensinfiltrated flowers relative to control 35S:GFP-infiltrated flowers revealed an increase in OfERF61 transcription levels in the former (Fig. 7a). The transcription of $N b C C D 4$ was affected by the overexpression of OfERF61, with a 1.8-fold increase in OfERF61-overexpressing petals compared with the control (Fig. 7b).

\section{Interaction of ERF61 with the CCD4 promoter}

Promoter analysis of $C C D 4$ revealed that the region upstream of the $C C D 4$ promoter contained several CAACA-binding elements (Supplementary Fig. 1), suggesting that $C C D 4$ is regulated by the AP2/ERF transcription factor. When the combination of $35 \mathrm{~S}_{\text {pro: }}$ : GFP and CCD $4_{\text {pro: }}$ GUS carrier (control) and the combination of $35 \mathrm{~S}_{\mathrm{pro}}$ :ERF61 and $\mathrm{CCD} 4_{\text {pro: }}$ GUS carrier were transformed into the tobacco leaves (Fig. 7a), the cultured and stained leaves appeared blue. However, the color of the leaves in the control group was lighter, whereas the color of the tobacco leaves overexpressing ERF61 was significantly deeper (Fig. 7c). qRT-PCR analysis of the tobacco leaves also indicated that GUS was significantly upregulated (3.37 times) in leaves that were cotransformed with $35 \mathrm{~S}_{\mathrm{pro}}$ :ERF61 and CCD4 $4_{\mathrm{pro}}$ : GUS carrier compared with the control group (Fig. 7d). In the yeast one-hybrid assay, the yeast cells that were cotransformed with pJG-ERF61 and the pLacZi-C1 carrier appeared blue after 20 min of staining, whereas the yeast cells in the control group, which were cotransformed with pJG and pLacZi-C1, did not appear blue, even after $8 \mathrm{~h}$ of staining (Fig. 7e). This suggests that ERF61 binds to the CCD4 promoter and thereby 

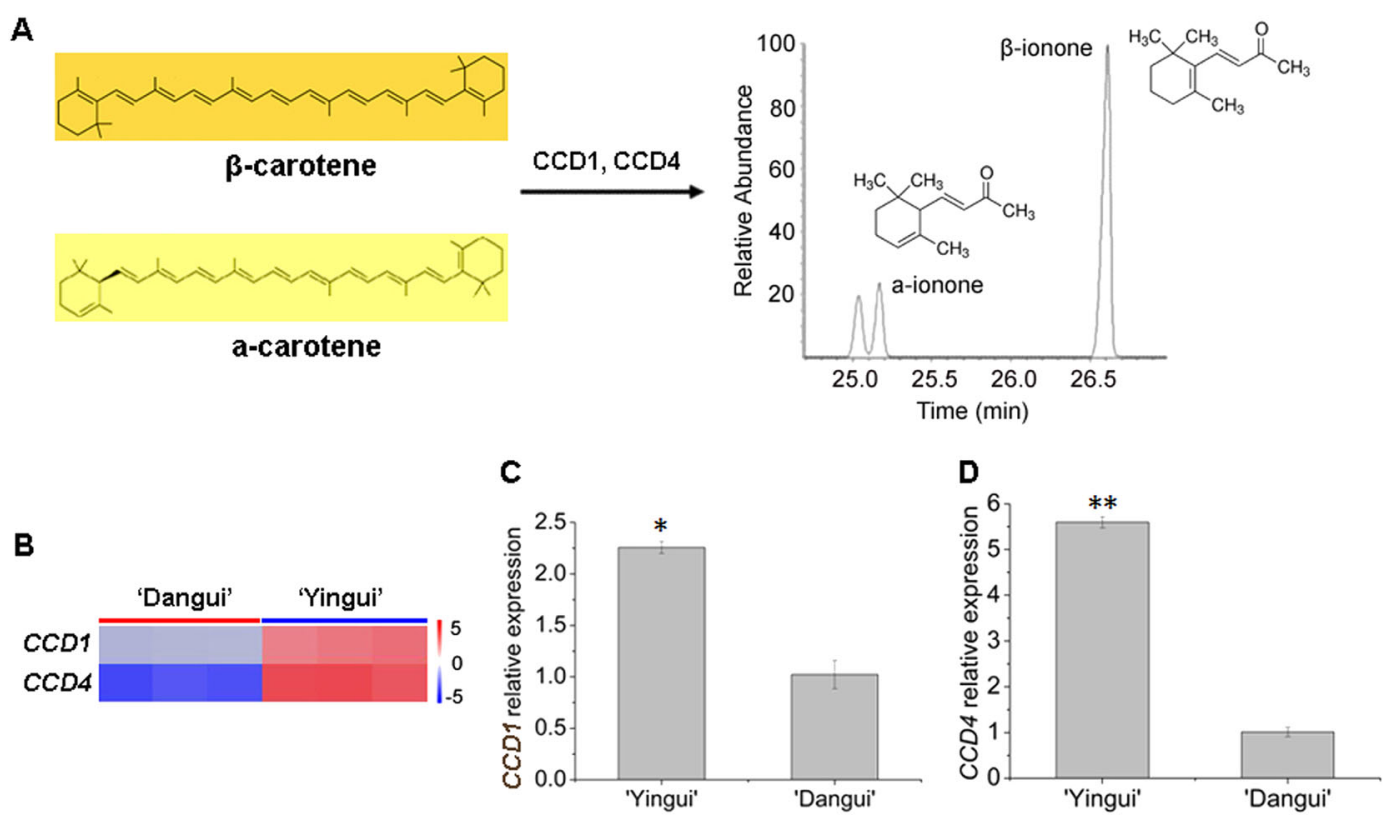

Fig. 5 Transcript levels of the CCD1 and CCD4 genes in the petals of 'Yingui' and 'Dangui' in the full flowering stage. a CCD1 and CCD4 can cleave $\mathbf{a}$-carotene and $\beta$-carotene into ionones; $\mathbf{b}$ CCD1 and CCD4 expression profiles from RNA-Seq; c qRT-PCR results for the CCD1 and CCD4 genes. The qRT-PCR data represent the means \pm SD of the three replicates from three independent experiments. ${ }^{*} P<0.05$, ${ }^{* *} P<0.01$
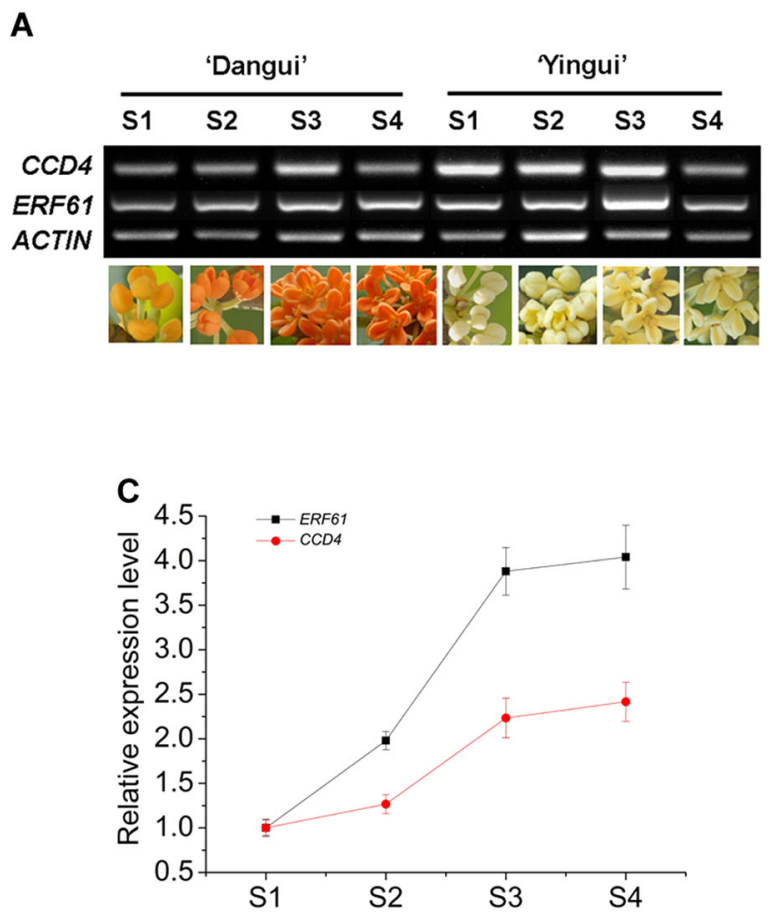

B
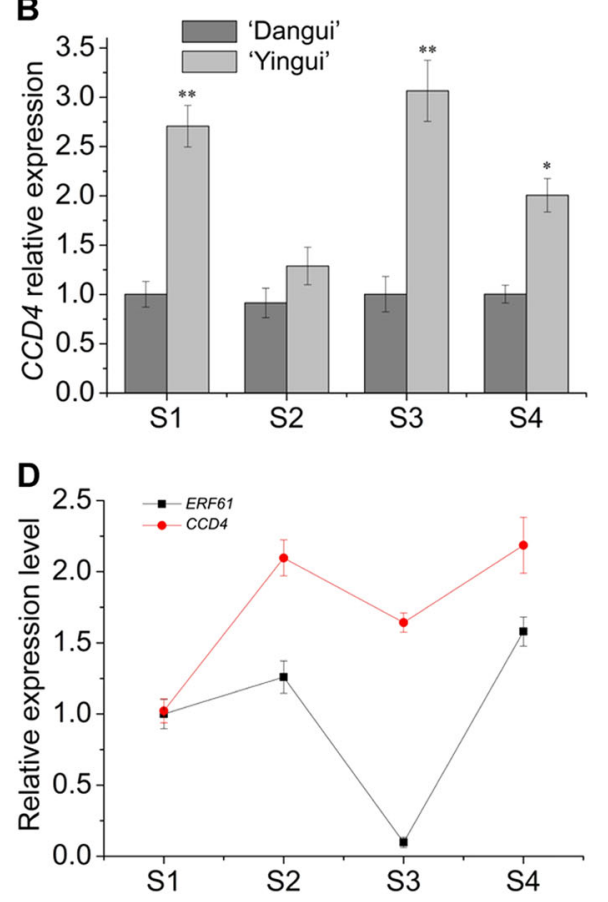

Fig. 6 RT-PCR and qRT-PCR analysis of ERF61 and CCD4 transcripts in different flowering stages of 'Dangui' and 'Yingui'. ACTIN transcripts were amplified as an internal control. a RT-PCR detection of ERF61 and CCD4 transcripts in different flowering stages of 'Dangui' and 'Yingui'; $\mathbf{b}$ qRTPCR analysis of CCD4 transcripts in different flowering stages of 'Dangui' and 'Yingui'; c qRT-PCR analysis of ERF61 and CCD4 transcripts in S1-S4 of 'Yingui'; $\mathbf{d}$ qRT-PCR analysis of ERF61 and CCD4 transcripts in S1-S4 of 'Dangui'. The qRT-PCR data represent the means \pm SD of the three replicates from three independent experiments. ${ }^{*} P<0.05,{ }^{*} P<0.01$ 


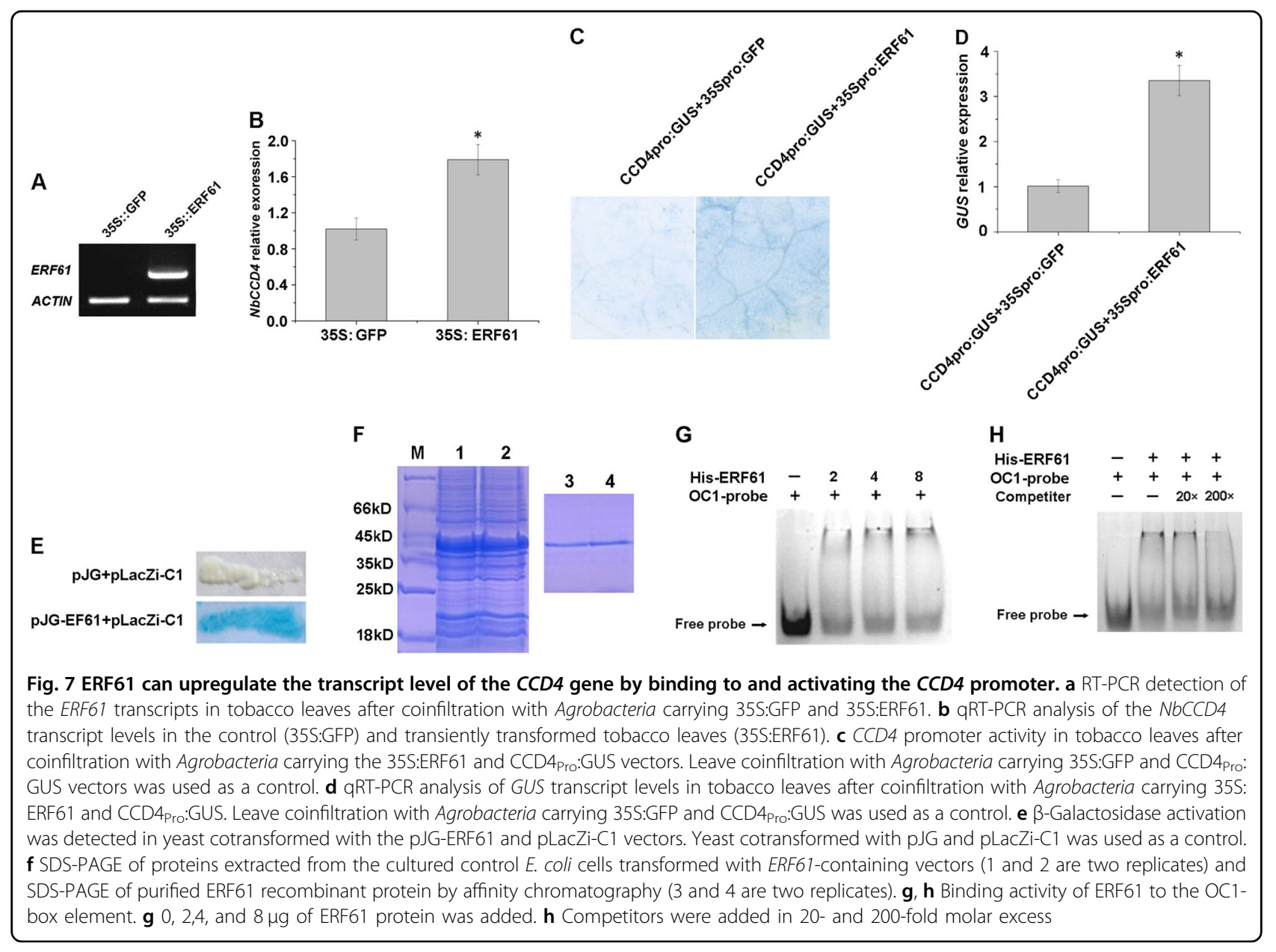

activates corresponding elements that increase GUS expression. The ERF61 protein was expressed in prokaryotes and purified (Fig. 7f). During EMSA, only one band was observed when the ERF61 protein was absent, whereas an obvious second band was observed when the OC1-labeled probe and ERF61 protein were added simultaneously. The additional band was the result of binding between the probe and the protein, and the amount of probe-protein complex gradually increased with increasing protein concentration (Fig. $7 \mathrm{~g}$ ). To verify the binding specificity of OfERF61, competition experiments were conducted by adding competitors (unlabeled OC1 probe) in 20- and 200-fold molar excess to the binding assay. The binding signals were reduced with the addition of 20-fold competitive unlabeled specific probe and not detected by adding 200 -fold competitive unlabeled probe (Fig. 7h). These results suggest that ERF61 acts as a transcription factor and directly binds to the CAACA element that is upstream of the $C C D 4$ promoter, thereby positively regulating $C C D 4$ expression.

\section{Discussion}

Previous studies have shown that the flower petals of sweet osmanthus contain many aromatic compounds, also called volatile oils ${ }^{11-13}$. The lipid granules in the plant cell can be dyed a grayish-black, brown-black, or black color using Sudan Black staining ${ }^{30}$. The present study used Sudan Black to stain sweet osmanthus petals. Both the epidermal and palisade cells of the petals were stained brown-black, and brown-black granules were also observed within the cells. This indicated that the sweet osmanthus petal cells contained large amounts of lipids, which could be related to the floral scent.

The synthesis of osmiophilic substances may involve the participation of Golgi bodies, the endoplasmic reticulum, plastids, and mitochondria. For example, the Golgi bodies and endoplasmic reticulum have been reported to participate in the synthesis of osmiophilic granules in the ciliated cells of Typhonium venosum (Araceae) ${ }^{9}$, and the present study revealed large numbers of osmiophilic granules in the petal cells of sweet osmanthus. The osmiophilic granules could be aggregates of aromatic 
substances. It is possible that such osmiophilic granules are transported outside the cell after synthesis and that the large numbers of osmiophilic granules observed on the cell walls may later be transported through cell gaps to the petal surface. This observation was confirmed by cryoSEM, which revealed large numbers of granules on epidermal cells, especially on brush-shaped hairs. However, further research is needed to clarify the mechanism by which osmiophilic granules are synthesized and transported to the petal surface.

ESEM and cryo-SEM revealed strips of brush-shaped hairs on the surfaces of the petals of the 'Dangui' and 'Yingui' cultivars. This result was also confirmed by cryo-SEM analysis for the other Dangui and Yingui group cultivars. However, ESEM indicated that the brush-shaped hairs were smooth and that granules were absent, whereas cryo-SEM indicated that large numbers of granules were present on the brush-shaped hairs. This difference may have resulted from the sample preparation for ESEM, since granules on the petal surfaces might have fallen off after vacuum drying. The granules are aggregates of aromatic substances secreted by the sweet osmanthus petals and are very volatile. The number of granules on the 'Yingui' petal surfaces was far higher than that on the 'Dangui' petal surfaces, possibly because more aromatic components were produced by the 'Yingui' petals or because the aromatic components in the 'Yingui' petals were more prone to aggregation. The GC-MS results indicated that 35 and 30 scent components were present in the 'Yingui' and 'Dangui' petals, respectively. Furthermore, the 'Yingui' petals contained more $\beta$-ionone, whereas the 'Dangui' petals contained more linalool compounds. Since the molecular weight, density, flash point, and boiling point of linalool are lower than those of $\alpha$-ionone and $\beta$-ionone (Table 1), linalool compounds tend to be more volatile, potentially resulting in fewer granules on the surface of 'Dangui' petals. However, the 'Yingui' petals contain more $\beta$-ionone, which is less volatile and tends to form granules.

Table 1 Chemical and physical properties of $\beta$-ionone, $\alpha$ ionone, and linalool

\begin{tabular}{llll}
\hline & B-lonone & a-lonone & Linalool \\
\hline CAS-No & $14901-07-6$ & $6901-97-9$ & $78-70-6$ \\
Exact mass & 192.15 & 192.15 & 154.25 \\
Boiling point $\left({ }^{\circ} \mathrm{C}\right)$ & 262.95 & 259.5 & 198.5 \\
Melting point $\left({ }^{\circ} \mathrm{C}\right)$ & 64.55 & 47.79 & 25 \\
Flash point $\left({ }^{\circ} \mathrm{C}\right)$ & 122 & 122 & 78 \\
\hline
\end{tabular}

The data from the SciFinder database
Most of the cells in sweet osmanthus petals are involved in the synthesis and secretion of aromatic substances and because they exhibit the characteristics of aromatic glands, these cells should be described accordingly. The entire sweet osmanthus petal can be considered a large aromatic gland since its basic tissues secrete aromatic substances that are then excreted by brush-shaped hairs on the cell surface.

Linalool synthase (LIS) uses geranyl pyrophosphate as a substrate and catalyzes the formation of linalool, a monoterpene alcohol with a sweet fragrance that occurs in floral scents of a wide variety of plants ${ }^{34-36}$. Constitutively high linalool emission has been engineered in several transgenic plant species by overexpressing the gene for $L I S^{37-40}$, which is a key enzyme in the synthesis of linalool and its oxidized product. In the present study, transcriptome sequencing revealed that LIS1 expression was significantly greater in the 'Dangui' petals than in the 'Yingui' petals (Fig. 4b), and qRT-PCR confirmed that the expression of LIS1 in the 'Dangui' petals was 5.1 times greater compared to that in the 'Yingui' petals (Fig. 4c). Furthermore, transcriptome sequencing revealed that MECS, HDR, and IDI1, all of which function upstream of monoterpenoid metabolism, were upregulated in the 'Dangui' petals (Fig. 4b), and qRT-PCR confirmed that the three genes were upregulated by 1.5-, 4.1-, and 2.2-fold, respectively (Fig. 4c). These results indicate that the upregulation of MECS, HDR, IDI1, and especially LIS1 ultimately increases the levels of linalools and oxidized linalools in the 'Dangui' petals to levels that are much higher than those present in the 'Yingui' petals.

Sweet osmanthus CCD1 can cleave $\alpha$-carotene into $\alpha$ ionone and $\beta$-ionone, whereas CCD4 can cleave $\beta$-carotene into $\beta$-ionone ${ }^{18-20,41}$. Both CCD1 and CCD4 are crucial for the cleavage of carotene into ionones. In the present study, the expression of both CCD1 and CCD4 in the 'Yingui' petals was significantly higher than that in the 'Dangui' petals, which indicated that the 'Yingui' petals should produce more $\alpha$-ionone and $\beta$-ionone, as confirmed by GC-MS analysis. CCD4 mainly cleaves $\beta$-carotene into $\beta$-ionone. In addition, the expression of $C C D 4$ in the 'Yingui' petals was significantly higher compared to that in 'Dangui', regardless of the flowering stage. Therefore, $C C D 4$ is a crucial contributor to the greater $\beta$-ionone content in the 'Yingui' petals.

Previous studies have suggested that ERF transcription factors function either directly or in cooperation with other proteins or bind to relevant gene promoters, thereby regulating terpenoid metabolism in plants ${ }^{42,43}$. In Artemisia annua, AaEFR1 and AaEFR2 have been reported to bind to CBF2 and RAV1AAT elements on the promoters of $A D S$ and $C Y P 71 A V 1$, respectively, thereby regulating the synthesis of artemisinin ${ }^{44}$. In C. roseus, the AP2/ERF transcription factors OCA3 and OCA4 bind to the 
promoters of TDC, STR, CPR, and other genes involved in the terpenoid indole alkaloid metabolic pathway ${ }^{45}$. In the present study, the expression of the cloned ERF transcription factor gene ERF61 was generally identical to that of CCD4 during the flowering stages, regardless of cultivar. The transcription of $N$. benthamiana CCD4 was affected by overexpression of OfERF61, with a 1.8-fold increase in OfERF61-overexpressing tobacco leaves compared to the control. Therefore, the ERF61 transcription factor may regulate the expression of $\mathrm{CCD} 4$. The tobacco transformation experiments revealed that ERF61 binds to the $C C D 4$ promoter, and the yeast one-hybrid and EMSA experiments revealed that ERF61 binds to the CAACA element in the $C C D 4$ promoter. Together, these results suggest that ERF61 regulates the expression of CCD4, thereby regulating the cleavage of carotenoids and synthesis of $\beta$-ionone in sweet osmanthus petals and ultimately regulating the floral scent of sweet osmanthus. In this study, we confirmed that $O f C C D 4$ was regulated by ERF61. The CCD4 gene may be regulated by other activator proteins. Future studies should endeavor to identify the regulating factors involved in the cleavage of carotenoids and further to elucidate the $\beta$-ionone biosynthesis machinery.

\section{Data Archiving Statement}

We have read and understood your journal's policies, and we believe that neither the manuscript nor the study violates any of these policies.

\section{Acknowledgements}

This research was supported by the National Natural Science Fund in China (Nos. U1604114 and 31400521).

\section{Conflict of interest}

The authors declare that they have no conflict of interest.

Supplementary Information accompanies this paper at (https://doi.org/ 10.1038/s41438-019-0189-4).

Received: 14 March 2019 Revised: 26 July 2019 Accepted: 29 July 2019 Published online: 07 September 2019

\section{References}

1. Maffei, M. E., Gertsch., J. \& Appendino, G. Plant volatiles: production, function and pharmacology. Nat. Prod. Rep. 28, 1359-1380 (2011).

2. Pichersky, E. \& Gershenzon, J. The formation and function of plant volatiles: perfumes for pollinator attraction and defense. Curr. Opin. Plant Biol. 5, 237-243 (2002).

3. Knudsen, J. T. Floral scents - a checklist of volatile compounds isolated by head-space techniques. Phytochemistry 33, 253-280 (1993).

4. Dudareva, N., Piechulla, B. \& Pichersky, E. Biogenesis of floral scents. Hortic. Rev. 24, 31-54 (2000).

5. Vogel, S. Duftdrüsen im Dienste der Bestäubung. Über Bau und Funktion der Osmophoren. Abh. der Math. Nat. Kl., Akad. der Wiss., Mainz 10, 1-165 (1962).

6. Hadacek, F. \& Weber, M. Club-shaped organs as additional osmophores within the Sauromatum inflorescence: odour analysis, ultrastructural changes and pollination aspects. Plant Biol. 4, 367-383 (2002).
7. Curry, K. J. Initiation of terpenoid synthesis in osmophores of Stanhopea anfracta (Orchidaceae): a cytochemical study. Am. J. Bot. 74, 1332-1338 (1987).

8. Curry, K. J., Mcdowell, L. M., Judd, W. S. \& Stern, M. L. Osmophores, floral features, and systematics of Stanhopea (Orchidaceae). Am. J. Bot. 78, 610-623 (1991).

9. Skubatz, H. \& Kunkel, D. D. Further studies of the glandular tissue of the Sauromatum guttatum (Araceae) appendix. Am. J. Bot. 86, 841-854 (1999).

10. Pridgeon, A. M. \& Stern, W. L. Ultrastructure of osmophores in Restrepia (Orchidaceae). Am. J. Bot. 70, 1233-1243 (1983).

11. Wang, L. M. et al. Variations in the components of Osmanthus fragrans Lour. essential oil at different stages of flowering. Food Chem. 114, 233-236 (2009).

12. Zeng, X. L. et al. Emission and accumulation of monoterpene and the key terpene synthase (TPS) associated with monoterpene biosynthesis in Osmanthus fragrans Lour. Front. Plant Sci. 6, 1232 (2016).

13. Yang, X. L. et al. The chromosome-level quality genome provides insights into the evolution of the biosynthesis genes for aroma compounds of Osmanthus fragrans. Hortic. Res. 5, 72 (2018).

14. Xiang, Q. B. \& Liu, Y. L. An Illustrated Monograph of the Sweet Osmanthus Variety in China 93-260 (Zhejiang Science \& Technology Press, Hangzhou, Zhejiang Province 2007)

15. He, Y. X. et al. The first genetic map in sweet Osmanthus (Osmanthus fragrans Lour) using specific locus amplified fragment sequencing. Front. Plant Sci. 8 , 1621 (2017)

16. Han, Y. J., Liu, L. X., Dong, M. F. \& Shang, F. D. CDNA cloning of the phytoene synthase (PSY) and expression analysis of PSY and carotenoid cleavage dioxygenase genes in Osmanthus fragrans. Biologia 68, 258-263 (2013).

17. Han, Y. J., Wang, X. H., Chen, W. C. \& Shang, F. D. Differential expression of carotenoid-related genes determines diversified carotenoid coloration in flower petal of Osmanthus fragrans. Tree Genet. Genomes 10, 329-338 (2014).

18. Baldermann, S. et al. Functional characterization of a carotenoid cleavage dioxygenase 1 and its relation to the carotenoid accumulation and volatile emission during the floral development of Osmanthus fragrans Lour. J. Exp. Bot. 61, 2967-2977 (2010).

19. Susanne, B. Biosynthesis of $\mathrm{a}$ - and $\beta$-ionone, prominent scent compounds, in flowers of Osmanthus fragrans. Acta Biochim. Pol. 59, 79-81 (2012).

20. Huang, F. C., Molnar, P. \& Schwab, W. Cloning and functional characterization of carotenoid cleavage dioxygenase 4 genes. J. Exp. Bot. 60, 3011-3022 (2009).

21. Han, Y. J., Wu, M., Cao, L. Y. \& Shang, F. D. Characterization of OfWRKY3, a transcription factor that positively regulates the carotenoid cleavage dioxygenase gene OfCCD4 in Osmanthus fragrans. Plant Mol. Biol. 91, 485-496 (2016).

22. Nakano, T., Suzuki, K., Fujimura, T. \& Shinshi, H. Genome wide analysis of the ERF gene family in Arabidopsis and rice. Plant Physiol. 140, 411-432 (2006).

23. Sakuma, Y. et al. DNA-binding specificity of the ERF/AP2 domain of Arabidopsis DREBs transcription factors involved in dehydration-and coldinducible gene expression. Biochem. Biophys. Res. Commun. 290, 998-1009 (2002).

24. Kagaya, Y., Ohmiya, K. \& Hattori, T. RAV1, a novel DNA-binding protein, binds to bipartite recognition sequence through two distinct DNA-binding domains uniquely found in higher plants. Nucleic Acids Res. 27, 470-478 (1999).

25. Sohn, K. H., Lee, S. C., Jung, H. W., Hong, J. K. \& Hwang, B. K. Expression and functional roles of the pepper pathogen-induced transcription factor RAV1 in bacterial disease resistance, and drought and salt stress tolerance. Plant Mol. Biol. 61, 897-915 (2006)

26. Boer, K. D. et al. Goossens A. Apetala2/ethylene response factor and basic elixloop-helix tobacco transcription factors cooperatively mediate jasmonateelicited nicotine biosynthesis. Plant J. 66, 1053-1065 (2011).

27. Fits, L. V. D. \& Memelink, J. The jasmonate-inducible AP2/ERF-domain transcription factor ORCA3 activates gene expression via interaction with a jasmonate-responsive promoter element. Plant J. 25, 43-53 (2001).

28. Han, Y. J. et al. CDNA-AFLP analysis on 2 Osmanthus fragrans cultivars with different flower color and molecular characteristics of OfMYB1 gene[]]. Trees 29, 931-940 (2015).

29. Han, S. et al. Mitogen-activated protein kinase 6 controls root growth in Arabidopsis by modulating $\mathrm{Ca}^{2+}$-based $\mathrm{Na}^{+}$flux in root cell under salt stress [J]. J. Plant Physiol. 171, 26-34 (2014). 
30. Karel, B. Detection of lipids in the plant meristematic cell with the aid of Sudan black staining. Biol. Plant 6, 142-151 (1964).

31. Bayliss, O. B. \& Adams, C. W. M. Bromine-sudan black: a general stain for lipids including free cholesterol. Histochem. J. 4, 505-515 (1972).

32. Khouri, M. R., Huang, G. \& Shiau, Y. F. Sudan stain of fecal fat; new insight into an old test. Gastroenterology 96, 421-427 (1989).

33. Thakur, M. S., Prapulla, S. G. \& Karanth, N. G. Microscopic observation of sudan black b staining to monitor lipid production by microbes. J. Chem. Technol. Biotechnol. 42, 129-134 (2007).

34. Cseke, L., Dudareva, N. \& Pichersky, E. Structure and evolution of Linaloo synthase. Mol. Biol. Evol. 15, 1491-1498 (1998).

35. Dudareva, N., Cseke, L., Blanc, V. M. \& Pichersky, E. Evolution of floral scent in Clarkia: novel patterns of S-linalool synthase gene expression in the C. breweri flower. Plant Cell 8, 1137-1148 (1996).

36. Kamatou, G. P. P. \& Viljoen, A. M. Linalool-a review of a biologically active compound of commercial importance. Nat. Prod. Commun. 3, 1183-1192 (2008).

37. Aharoni, A. et al. Terpenoid metabolism in wild-type and transgenic Arabidopsis plants. Plant Cell 15, 2866-2884 (2003)

38. Aharoni, $\mathrm{A}$. et al. Metabolic engineering of terpenoid biosynthesis in plants. Phytochem. Rev. 5, 49-58 (2006).
39. Lavy, M. et al. Linalool and linalool oxide production in transgenic carnation flowers expressing the Clarkia breweri linalool synthase gene. Mol. Breed. 9, 103-111 (2002).

40. Lewinsohn, E. et al. Enhanced levels of the aroma and flavor compound Slinalool by metabolic engineering of the terpenoid pathway in tomato fruits. Plant Physiol. 127, 1256-1265 (2001).

41. Zhang, $X$. et al. Overexpression and characterization of CCD4 from Osmanthus fragrans and $\beta$-ionone biosynthesis from $\beta$-carotene in vitro. J. Mol. Catal. B Enzymatic 134, 105-114 (2016).

42. Licausi, F., Ohme-Takagi, M. \& Perata, P. APETALA2/ethylene responsive factor (AP2/ERF) transcription factors: mediators of stress responses and developmental programs. New Phytol. 199, 639-649 (2013).

43. Liu, J. Q. et al. A novel AP2/ERF transcription factor CR1 regulates the accumulation of vindoline and serpentine in Catharanthus roseus. Front. Plant Sci. 8 , 2082 (2017).

44. Yu, Z. X. et al. The jasmonate-responsive AP2/ERF transcription factors AaERF1 and AaERF2 positively regulate artemisinin biosynthesis in Artemisia annua $\mathrm{L}$ Mol. Plant 5, 353-365 (2012).

45. Paul, P. et al. A differentially regulated AP2/ERF transcription factor gene cluster acts downstream of a MAP kinase cascade to modulate terpenoid indole alkaloid biosynthesis in Catharanthus roseus. New Phytol. 213, 1107-1123 (2017). 